\title{
Expression and Localization of Testis Developmental Related Gene 1 (TDRG1) in Human Spermatozoa
}

\author{
Houyang Chen, ${ }^{1, *}$ Jie Sun, ${ }^{1, *}$ Yuanqiao $\mathrm{He}^{2}$, Qianxing Zou, ${ }^{2}$ Qiongfang $\mathrm{Wu}^{1}$ and \\ Yuxin Tang ${ }^{3}$ \\ ${ }^{1}$ Reproductive Medical Center, Jiangxi Provincial Maternal and Child Health Hospital, Nanchang, Jiangxi, P.R. \\ China \\ ${ }^{2}$ Institute of Life Science, Nanchang University, Nanchang, Jiangxi, P.R. China \\ ${ }^{3}$ Department of Andrology, The Third Xiangya Hospital of Central South University, Changsha, Hunan, P.R. \\ China
}

\begin{abstract}
Testis-specific proteins, synthesized during spermatogenesis and spermiogenesis, are necessary for spermatid differentiation and/or for mature sperm function during fertilization. However, majority of these genes have neither been identified nor fully characterized. Testis developmental related gene 1 (TDRG1), a newly identified human testis-specific gene, encodes a 100-amino-acid protein without any characterized protein domains, and it may play a role in spermatogenesis. However, whether this human-specific protein is important for mature sperm function remains unclear. As an initial effort, in this study, we aimed to systematically investigate the expression and localization of TDRG1 in normal human spermatozoa. Thus, immunohistochemistry was used to analyze the distribution of TDRG1 in human testis. Reverse transcription-polymerase chain reaction, western blot analysis and indirect immunofluorescence were used to determine the expression and localization of TDRG1 in normal human spermatozoa. The immunohistochemistry results showed that the TDRG1 protein was expressed in spermatogenic cells in the seminiferous tubules of human testis. Interestingly, the TDRG1 was more abundant in spermatogenic cells at the late stages of spermatogenesis. The TDRG1 antibody specifically recognized an 11-kDa protein only in soluble extracts from normal human spermatozoa. Indirect immunofluorescence assays indicated that TDRG1 located in the midpiece, principal piece and flagellum of normal human spermatozoa. In conclusion, TDRG1 was found not only in spermatogonia, but also in spermatozoa. The localization of TDRG1 in human normal spermatozoa implies its potential regulatory role in sperm motility.
\end{abstract}

Keywords: human spermatozoa; immunofluorescence; sperm motility; spermatogenesis; testis developmental related gene 1

Tohoku J. Exp. Med., 2015 February, 235 (2), 103-109. ㄷ 2015 Tohoku University Medical Press

\section{Introduction}

Spermatogenesis is a complex biological process involving the mitotic proliferation of spermatogonia, the meiotic division of spermatocytes, and the morphogenic differentiation of spermatids to mature spermatozoa. During spermiogenesis, the round spermatids undergo dramatic morphological and cellular changes, including formation of the acrosome, elongation and condensation of the nucleus, formation of the flagellum, and disposal of unnecessary cytoplasm, to produce highly specialized and polarized spermatozoa (Hecht 1998). Though many genes have been discovered and confirmed to be related to spermato- genesis (Liu et al. 2004; Mu et al. 2006; Yang et al. 2011b), there are still many unidentified and uncharacterized genes and, most importantly, it remains to be clarified how spermatogenesis is controlled and regulated by these genes and their interactions (Eddy 2002; Poongothai et al. 2009; Zheng et al. 2010). Thus, further research on novel genes and investigation of their functions is crucial to help us elucidate the mechanisms of spermatogenesis and the underlying gene network.

Taking advantage of a computerized program, digital differential display (Scheurle et al. 2000; Olesen et al. 2001), previously Jiang and collaborators (Jiang et al. 2011) compared the differences of gene expression profiles among

Received December 24, 2014; revised and accepted January 27, 2015. Published online February 6, 2015; doi: 10.1620/tjem.235.103.

*These two authors contributed equally to this work.

Correspondence: Qiongfang Wu, Reproductive Medical Center, Jiangxi Provincial Maternal and Child Health Hospital, Nanchang, Jiangxi 330006, P.R. China.

e-mail: wuqiongfang898@sina.com

Yuxin Tang, Department of Andrology, The Third Xiangya Hospital of Central South University, Changsha, Hunan 410013, China.

e-mail: mmcct@126.com 
cDNA libraries from various human tissues, and cloned a novel human testis-specific gene, testis developmental related gene 1 (TDRG1), which is located in chromosome 6 (Peng et al. 2012). Expression of TDRG1 was found only in human testicular tissue, indicating that it is a unique gene in human germ cells. As reported previously, the germ cellspecific and development-regulated genes underlying the intrinsic genetic network are the primary regulators of spermatogenesis (Eddy 2002). TDRG1 is mainly located in spermatogenic cells in the seminiferous tubules of adult testis. The expression of TDRG1 exhibits highest level in human pubertal testis, then shows an age-dependent decrease. Obviously, TDRG1 is related to the function of spermatogenesis (Jiang et al. 2011). Because spermatozoa are transcriptionally and translationally inactive, several sperm proteins synthesized in the haploid spermatid will remain in the morphologically mature sperm after it leaves the testis. Therefore, proteins that are synthesized during spermatogenesis and spermiogenesis are necessary for spermatid differentiation and/or for sperm function during fertilization. It has been reported that some proteins are specifically expressed during the spermatogenic process and then located in spermatozoa (Visconti et al. 2001; Li et al. 2002; Hao et al. 2004; Li et al. 2006). In the case of TDRG1, although its specific expression in testis has been identified (Jiang et al. 2011), the distribution profile and possible function of TDRG1 in human spermatozoa remains unclear.

In this study, we analyzed the distribution of TDRG1 in human testis and determined the expression and localization of TDRG1 in normal human spermatozoa using reverse transcription-polymerase chain reaction (RT-PCR), western blot analysis, and indirect immunofluorescence. The aim of the present study was to systematically investigate the expression profile and localization of TDRG1 in normal human spermatozoa.

\section{Materials and Methods}

The sample collection was approved by the Institutional Ethics Committee on Human Subjects of Jiangxi Provincial Maternal and Child Health Hospital (China). The participants signed a consent form permitting use of their samples in the study. Specimens from various tissues of mature healthy human (25 40 years old) were obtained at autopsies from individuals who died from motor vehicle collisions. All chemicals and reagents used in this study were of molecular biology grade and purchased from Sigma-Aldrich (St. Louis, MO, USA) unless stated otherwise.

\section{Immunohistochemistry}

Human fertile testis specimens were obtained from biopsies, which were fixed in formalin and embedded in paraffin. Immunohistochemistry was performed as previously described (Wen et al. 2010). Briefly, anti-TDRG1 monoclonal antibody 10B6 (Jiang et al. 2011) (1:200) was used as the primary antibody. Biotinconjugated goat anti-rabbit IgG was used as the secondary antibody. 3,3-diaminobenzidine (Zhongshan Goldenbridge Biotechnology, Beijing, China) generated brown color at the site of peroxidase activ- ity. In the negative control slides, the primary antibody was replaced by goat serum without peptide immunization. The results were observed under a microscope (DMLB; Leica Microsystems, Wetzlar, Germany).

\section{Semen preparation}

Routine semen analysis monitored by a computer-assisted sperm analysis system (WLJY-9000; WeiLi Co., Ltd., Beijing, China) revealed that the spermatozoa were within the normal range stipulated by the guidelines (World Health Organization 2010). Freshly ejaculated, human semen samples were obtained at the Reproductive Medical Center, Jiangxi Provincial Maternal and Child Health Hospital from donors who are healthy and had proven fertility and practiced 2-7 days of sexual abstinence. The mean age of the 10 donors was $30.31 \pm 2.33$. To separate highly motile and morphologically normal spermatozoa from the semen, the liquefied ejaculate was washed on a two-layer ( $90 \%$ and $50 \%$ ) discontinuous gradient (Isolate; Irvine Scientific, Santa Ana, CA, USA) according to a modified protocol described previously (Makkar et al. 1999; Sharma et al. 1999). The morphology of purified spermatozoa was evaluated by Diff-Quik staining kit (Baso Diagnostics Inc., Zhuhai, China), and samples with more than $10 \%$ normal morphology sperm were used for subsequent experiments.

\section{Reverse transcription-polymerase chain reaction (RT-PCR)}

RT-PCR was performed according to a previously published method (Jiang et al. 2011). Briefly, total RNA from human testis, purified spermatozoa, and skeletal muscle was extracted with TRIzol Reagent (Invitrogen, Carlsbad, CA, USA), followed by treated with DNAaseI (Promega, Madison, WI, USA), and then reverse transcribed into cDNA in a final volume of $10 \mu \mathrm{L}$ with the RevertAid M-MuLV Reverse Transcription System (Fermentas, Glen Burnie, MD, USA). The PCR reaction was performed using Taq PCR Mastermix (Tiangen Biotech, Beijing, China) with the following primers: TDRG1(forward primer, 5'-AAGAGGAGGGAG GCAGTCT-3'; reverse primer, 5'-GCCCAATTCCTCTTGACT GA-3') and glyceraldehyde-3-phosphate dehydrogenase (GAPDH), as a positive control (forward primer, 5'-CCACCCATGGCAAAT TCCATGGCA-3'; reverse primer, 5'-TCTAGACGGCAGGTCAGG TCACC- $\left.3^{\prime}\right)$. The PCR programs used were: $95^{\circ} \mathrm{C}$ for $1 \mathrm{~min}$, followed by 35 cycles of $95^{\circ} \mathrm{C}$ for $15 \mathrm{~s}, 57^{\circ} \mathrm{C}$ for $15 \mathrm{~s}$, and $72^{\circ} \mathrm{C}$ for $45 \mathrm{~s}$ (GAPDH: $55^{\circ} \mathrm{C}, 25$ cycles), with a final extension at $72^{\circ} \mathrm{C}$ for $5 \mathrm{~min}$. The PCR products for TDRG1 and GAPDH were analyzed using a Rapid Agarose Gel Electrophoresis System (WealtecCorp, Sparks, $\mathrm{NV}, \mathrm{USA}$ ) in $1.5 \%$ agarose gels.

\section{Protein extraction}

The human testis and skeletal muscle protein extraction was performed according to the Tissue Protein Extraction Kit protocol (CWBio, Beijing, China). Total protein of human sperm was isolated according to a previously published method (Yang et al. 2011a). Briefly, human sperm were first lysed in lysis buffer $(50 \mathrm{mM} \mathrm{Na}$ phosphate, $150 \mathrm{mM} \mathrm{NaCl}, 10 \mathrm{mM} \mathrm{KCl}, \mathrm{pH}$ 7.2) with Protease Inhibitor Mixture (Sigma-Aldrich). Then, an equal volume of detergent solution ( $50 \mathrm{mM}$ Na phosphate, $150 \mathrm{mM} \mathrm{NaCl}, 10 \mathrm{mM} \mathrm{KCl}, 2 \%$ Triton X-100, 1\% Nadeoxycholate, $0.2 \%$ SDS, pH 7.2) was added to the lysed sperm suspension for membrane protein solubilization. The mixture was centrifuged by $12,000 \times \mathrm{g}$ at $4{ }^{\circ} \mathrm{C}$ and the protein concentration in the supernatant was determined by the bicinchoninic acid 
method (Thermo Scientific, Waltham, MA, USA). The extraction of sperm membrane protein was performed according to the manual of Mem-PER Eukaryotic Membrane Protein Extraction Kit (Thermo Fisher Scientific Inc., Rockford, IL, USA).

\section{Western blot analysis}

Approximately $50 \mu \mathrm{g}$ of protein were separated by $10 \%$ polyacrylamide gel electrophoresis and transferred onto polyvinylidenedifluoride membranes (Millipore Corp., Bedford, MA, USA). Membranes were blocked with 5\% nonfat milk in Tris-buffered saline for $2 \mathrm{~h}$ before incubation with the primary antibody (anti-TDRG1 monoclonal antibody 10B6; CatSper 3 polyclonal antibody, Abcam, Cambridge, UK; GAPDH polyclonal antibody, Thermo Scientific) overnight at $4{ }^{\circ} \mathrm{C}$. After washing, the membranes were probed with horseradish peroxidase-conjugated goat anti-mouse IgG (Thermo Scientific) at $37^{\circ} \mathrm{C}$ for $1 \mathrm{~h}$. An ECL detection kit (Thermo Scientific) was used to detect the peroxidase activity.

\section{Indirect immunofluorescence}

Indirect immunofluorescence assay was performed according to previously published method (Luo et al. 2015). Briefly, human sperm were fixed in $4 \%$ paraformaldehyde for $20 \mathrm{~min}$ on ice and rinsed with phosphate-buffered saline. The washed sperm were attached to ployL-lysine-coated coverslips and blocked in 5\% bovine serum albumin with $0.5 \%$ Triton X-100 (for permeabilizing the sperm) or without
$0.5 \%$ Triton X-100 (non-permeabilizing the sperm, Tubbs and Thomas 2009) for $2 \mathrm{~h}$. After blocking, the sperm were incubated with the primary antibody (anti-TDRG1 mouse monoclonal antibody 10B6, 1:100; Catsper3 rabbit polyclonal antibody, 1:100), overnight at $4^{\circ} \mathrm{C}$. Subsequently, the sperm were stained with DyLight 488 AffiniPure goat anti-mouse IgG (1:200) or DyLight 488 AffiniPure goat anti-rabbit IgG (1:200) (EarthOx Life Science, Millbrae, CA, USA) and $1 \mu \mathrm{M}$ DAPI. In the negative control, the primary antibody was replaced by goat serum without peptide immunization. After washing, the coverslips containing the stained sperms were loaded on a clear slide and examined under an FV1000-IX81 confocal laser scanning biological microscope (Olympus, Tokyo, Japan) with an LD laser (405) for the DAPI and an M_Ar laser (488 nm) for the Dylight 488.

\section{Results}

\section{The distribution of TDRG1 in human testis}

Using immunohistochemistry we examined the cellular distribution of TDRG1 protein in human biopsy testicular tissues. We found that TDRG1 was expressed in the cytoplasm of spermatogenic cells in the seminiferous tubules of adult normal human testis, which is consistent with previous reports (Jiang et al. 2011). Interestingly, TDRG1 was more abundant in spermatogenic cells of the
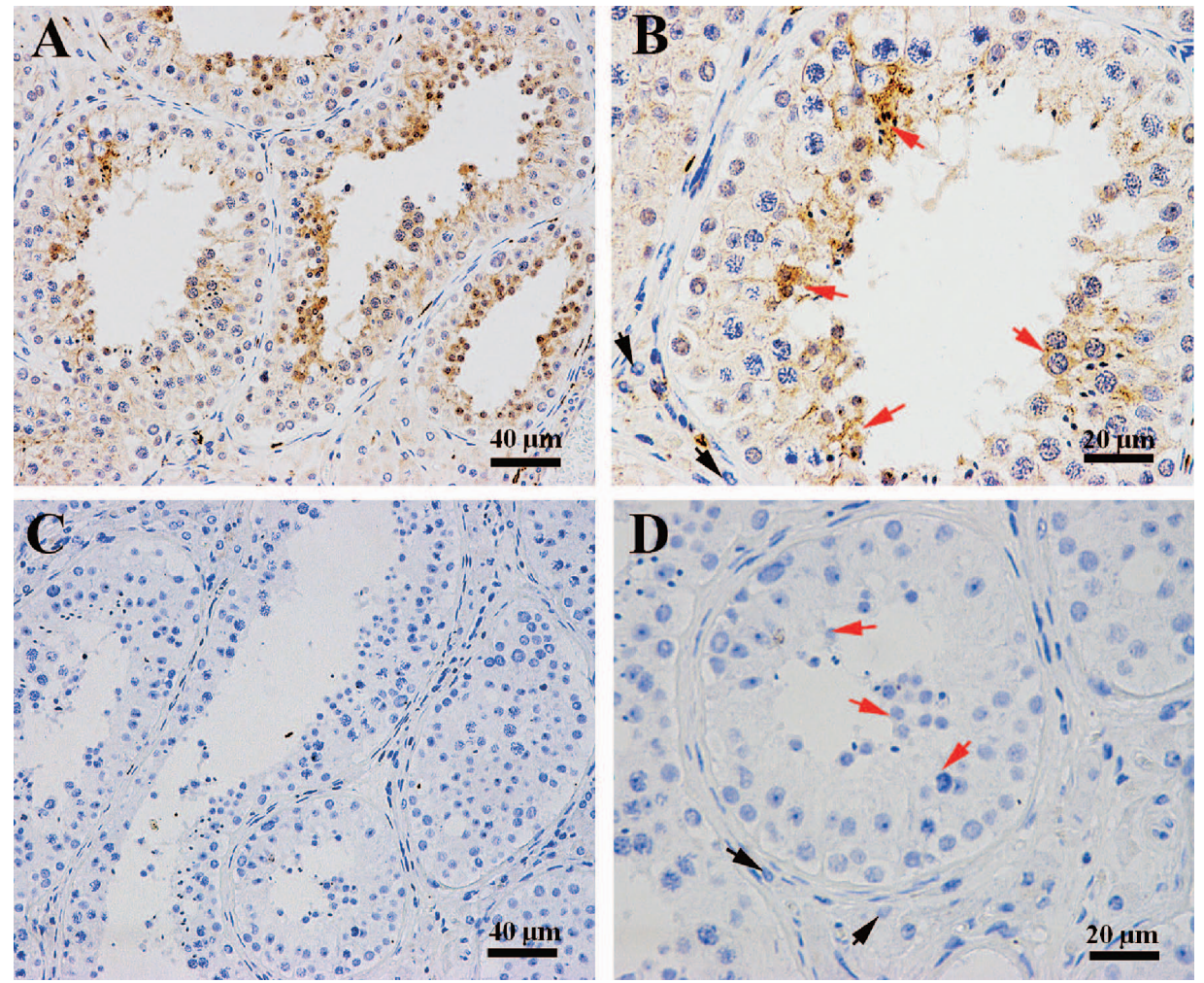

Fig. 1. Immunohistochemical distribution of TDRG1 in human testis.

Testes were probed with anti-TDRG1 monoclonal antibody 10B6. TDRG1 is predominately expressed in spermatogenic cells (shown in red arrows), but not in Leydig cells (shown in black arrows) in seminiferous tubules of adult testis. Furthermore, the TDRG1 was more abundant in spermatogenic cells of the late stages of spermatogenesis (A, B). The brown cells are TDRG1-positive cells. No staining (brown) was observed in tissue sections when the primary antibody was replaced by goat serum without peptide immunization as the negative control (C, D). Each experiment was repeated three times. A representative experiment was shown $(n=4)$. 
late stages of spermatogenesis (Fig. 1A, B), which suggest that TDRG1 may be involved in spermatogenesis. But whether TDRG1 plays a role in human spermatozoa remains unclear and need to be further elucidated. In contrast, almost no staining appeared in the control experiments (Fig. 1C, D).

\section{Expression of TDRG1 mRNA and protein in human testis and spermatozoa}

Encouraged by the result of immunohistochemistry, we further examined whether TDRG1 is expressed in human mature sperm. Semen samples from normozoospermic men were fractioned on a 2-layer discontinuous gradient, which was performed to separate normal spermatozoa from the semen. This sperm separation technique could effectively acquire sperm populations with high motility and normal morphology free of seminal plasma, debris, microbial contamination, and somatic and immature germ cells (Makkar et al. 1999; Sharma et al. 1999). It is very important for the following experiments. To this end, we collected 10 normal human sperm samples that were mixed into a pool after purification, and their quality was confirmed using the Diff-Quick method (Fig. 2A). Total RNA and proteins were extracted from the sperm to examine the TDRG1 expression. The RT-PCR results showed that the mRNA of TDRG1 was expressed in human spermatozoa and testis (Fig. 2B). A specific band with a molecular weight of approximate $11 \mathrm{kDa}$ was detected in human spermatozoa and testis, but not in skeletal muscle, by western blot analysis (Fig. 2C), which is consistent with previous reports (Jiang et al. 2011). These results indicate that TDRG1 also exists in human mature spermatozoa. Considering our immunohistochemistry evidence that TDRG1 is expressed in the cytoplasm of spermatogenic cells, we further examined whether TDRG1 is a cytoplasmic protein in human spermatozoa. The soluble and membrane proteins were separated from human spermatozoa to determine the distribution of TDRG1 in human spermatozoa. The results showed that TDRG1 was only detected as a soluble protein of human sperm (Fig. 2D). The cytoplas-
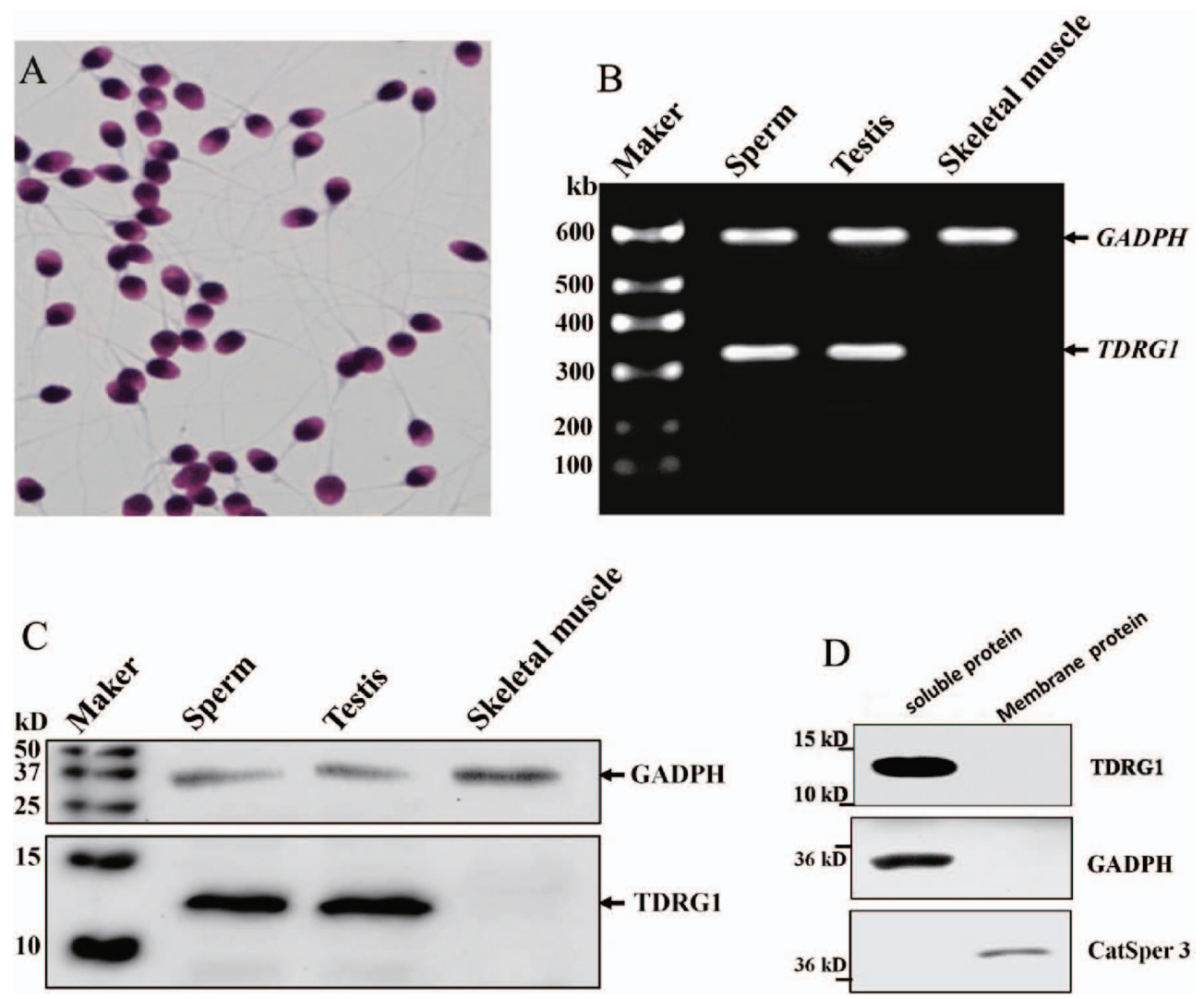

Fig. 2. Expression of TDRG1 mRNA and protein in human spermatozoa.

A. The morphology of purified spermatozoa was evaluated by Diff-Quik staining kit. The result showed normal morphology of human sperm without the contamination of testicular germ cells, leukocytes, or epithelial cells. B. TDRG1 mRNA was expressed in human sperm and testis but not in skeletal muscle by RT-PCR. The PCR products for TDRG1 and GAPDH were $320 \mathrm{bp}$ and $600 \mathrm{bp}$, respectively. C. TDRG1 protein was expressed in human sperm and testis but not in skeletal muscle by western blot analysis. GAPDH was used as internal standard. A band of $11 \mathrm{kDa}$ for TDRG1 and a band of $36 \mathrm{kDa}$ for GAPDH were detected. D. Determination of the TDRG1 protein in membrane proteins and soluble proteins extracted from human spermatozoa. Cytoplasmic protein GADPH and membrane protein CatSper 3 were used as the control. A band of $40 \mathrm{kDa}$ for CatSper3 was detected. Each experiment was repeated three times. A representative experiment was shown $(n=10)$. 
mic protein, GAPDH, and the membrane proteins, CatSper 3 , were also tested, confirming that there was no cross contamination between the two fractions (Fig. 2D).

\section{Localization of TDRG1 in human spermatozoa}

The functional abilities of a protein are largely dependent on its cellular localization (Anwer et al. 2014). Therefore, we further studied the localization of TDRG1 in spermatozoa. Based on our results that TDRG1 is a cytoplasmic protein, we used Triton X-100 to permeate the human sperm membrane. The results showed that TDRG1 protein located in the midpiece, principal piece and flagellum of human spermatozoa, but no fluorescence was detected in the head of the sperm (Fig. 3A), suggesting that its function may be associated with sperm motility. In contrast, there was no significant fluorescence in the non-permeated sperm, which was not treated with Triton X-100
(Fig. 3B). As a positive control, significant fluorescence of the membrane protein CatSper could be detected in the nonpermeate sperm (Fig. 3C). Overall, our results strongly support the idea that TDRG1 is a cytosolic protein in human sperm.

\section{Discussion}

TDRG1 is a human testis-specific gene that encodes a protein of 100 amino acids and contains no protein domains characterized in the Conserved Domain Database. As reported previously (Jiang et al. 2011), this novel protein has been proposed to have a functional role in spermatogenesis. However, the expression and localization of TDRG1 in human spermatozoa have not been reported. In our current study, we systematically studied expression profiles of TDRG1 in normal human spermatozoa. Immunohistochemical analysis showed that TDRG1 was expressed
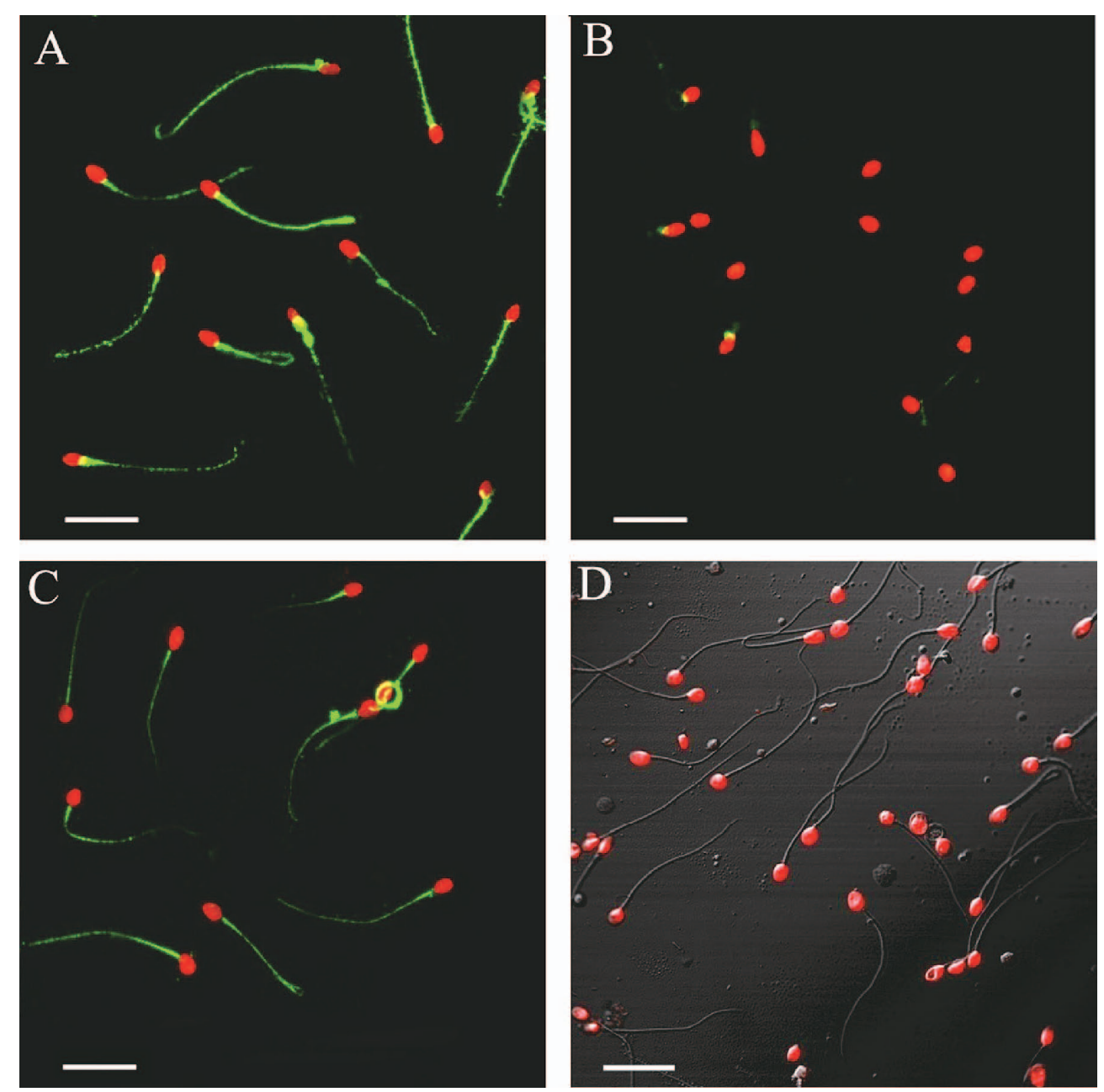

Fig. 3. Localization of TDRG1 protein in human spermatozoa by indirect immunofluorescence.

Permeabilized (A) and non-permeabilized (B) sperm were incubated with the anti-TDRG1 monoclonal antibody (1:100) and an DyLight 488 AffiniPure goat anti-mouse IgG (1:200) and visualized using an FV1000-IX81 confocal laser scanning biological microscope, respectively. Immunofluorescence localization of TDRG1 protein (green) and DAPI (red) was shown. The significant fluorescence of the membrane protein CatSper 3 was detected in non-permeabilized sperm (C). No fluorescence was observed in human spermatozoa when the primary antibody was replaced by goat serum as the negative control (D). Bars represent $20 \mu \mathrm{m}$. Each experiment was repeated three times. A representative experiment is shown $(n=10)$. 
in the cytoplasm of spermatogenic cells in the seminiferous tubules of adult normal human testis, which is consistent with a previous report (Jiang et al. 2011). In this study, immunohistochemical analysis further showed that TDRG1 is more abundant in spermatogenic cells of the late stage during spermatogenesis (Fig. 1). This result suggests that TDRG1 might be also present in human spermatozoa. Our further results of RT-PCR and Western blot analyses provided the evidence that TDRG1 is expressed in human spermatozoa. An 11-kDa protein in the soluble fraction of normal spermatozoa is regarded as the TDRG1 protein. These results suggest that TDRG1 may play a potential role in the human spermatozoa.

The cellular localization of TDRG1 in normal human spermatozoa was determined by indirect immunofluorescence, which is the first step toward our understanding of the role of these signaling molecules in the regulation of sperm functions. The results showed that TDRG1 was specifically located in the midpiece, principal piece and flagellum of human spermatozoa. The midpiece of sperm contains mainly mitochondrial sheath. Sperm mitochondrial functionality is physiologically relevant and needs to be maintained during the period between epididymal storage, ejaculation, and traveling in the female reproductive tract, until it reaches sperm-oocyte interactions. The expression of several mitochondrial proteins seems to be altered in sperm with low motility (Siva et al. 2010; Parte et al. 2012). It is known that mitochondria constitute major $\mathrm{Ca}^{2+}$ stores (Perl et al. 2006). The functional interaction of stored $\mathrm{Ca}^{2+}$ at the sperm neck/midpiece regulates the activities of CatSper channels in the principal piece (Costello et al. 2009). It has been reported that the principal piece of human sperm have some ion channels, such as potassium channels, sodium channels, calcium channels, which play an important role in sperm maturation, motility, capacitation, and sperm-egg binding (Cejudo-Roman et al. 2013; Zeng et al. 2013). TDRG1 protein could not be detected in non-permeabilized human spermatozoa membrane by indirect immunofluorescence, or in the sperm membrane proteins by western blot analysis, demonstrating that this form of TDRG1 protein is expressed in the cytoplasm of human spermatozoa. TDRG1 may not be a channel protein. The sperm flagellum is the specialized motile powerhouse of the cell and shares many similarities with cilia (Vadnais et al. 2014). The sperm flagellum is a complex structure whose integrity is essential for sperm motility and fertilization of the egg. These results hint that TDRG1 may be associated with the physiological function of human sperm, such as modulation of motility.

In conclusion, TDRG1 was found not only in spermatogonia, but also in mature spermatozoa, suggesting that TDRG1 may be synthesized during the entire stage of spermatogenesis. The localization of TDRG1 in human normal spermatozoa implicates that it may be related to human sperm motility. Nevertheless, further functional studies are required to define the specific roles of TDRG1 in human spermatozoa together with its involvement in male infertility.

\section{Acknowledgments}

We are very grateful to Prof. Zeng Xuhui and Dr. Luo Tao in the Institute of Life Science, Nanchang University for their excellent technical assistances and advice. This study was supported by the General Science and Technology Program of Health and Family Planning Commission of Jiangxi Province (Grant No. 20151119).

\section{Conflict of Interest}

The authors declare no conflict of interest.

\section{References}

Anwer, M.U., Boikoglou, E., Herrero, E., Hallstein, M., Davis, A.M., Velikkakam James, G., Nagy, F. \& Davis, S.J. (2014) Natural variation reveals that intracellular distribution of ELF3 protein is associated with function in the circadian clock. Elife, 3, e02206.

Cejudo-Roman, A., Pinto, F.M., Subiran, N., Ravina, C.G., Fernandez-Sanchez, M., Perez-Hernandez, N., Perez, R., Pacheco, A., Irazusta, J. \& Candenas, L. (2013) The voltagegated sodium channel nav1.8 is expressed in human sperm. PLoS One, 8, e76084.

Costello, S., Michelangeli, F., Nash, K., Lefievre, L., Morris, J., Machado-Oliveira, G., Barratt, C., Kirkman-Brown, J. \& Publicover, S. (2009) $\mathrm{Ca}^{2+}$-stores in sperm: their identities and functions. Reproduction, 138, 425-437.

Eddy, E.M. (2002) Male germ cell gene expression. Recent Prog. Horm. Res., 57, 103-128.

Hao, Z., Jha, K.N., Kim, Y.H., Vemuganti, S., Westbrook, V.A., Chertihin, O., Markgraf, K., Flickinger, C.J., Coppola, M., Herr, J.C. \& Visconti, P.E. (2004) Expression analysis of the human testis-specific serine/threonine kinase (TSSK) homologues. A TSSK member is present in the equatorial segment of human sperm. Mol. Hum. Reprod., 10, 433-444.

Hecht, N.B. (1998) Molecular mechanisms of male germ cell differentiation. Bioessays, 20, 555-561.

Jiang, X., Li, D., Yang, J., Wen, J., Chen, H., Xiao, X., Dai, Y., Yang, J. \& Tang, Y. (2011) Characterization of a novel human testis-specific gene: testis developmental related gene 1 (TDRG1). Tohoku J. Exp. Med., 225, 311-318.

Li, H.G., Liao, A.H., Ding, X.F., Zhou, H. \& Xiong, C.L. (2006) The expression and significance of CATSPER 1 in human testis and ejaculated spermatozoa. Asian J. Androl., 8, 301-306.

Li, T., Vu, T.H., Lee, K.O., Yang, Y., Nguyen, C.V., Bui, H.Q., Zeng, Z.L., Nguyen, B.T., Hu, J.F., Murphy, S.K., Jirtle, R.L. \& Hoffman, A.R. (2002) An imprinted PEG1/MEST antisense expressed predominantly in human testis and in mature spermatozoa. J. Biol. Chem., 277, 13518-13527.

Liu, S.F., Lu, G.X., Liu, G., Xing, X.W., Li, L.Y. \& Wang, Z. (2004) Cloning of a full-length cDNA of human testis-specific spermatogenic cell apoptosis inhibitor TSARG2 as a candidate oncogene. Biochem. Biophys. Res. Commun., 319, 32-40.

Luo, T., Li, N., He, Y.Q., Weng, S.Q., Wang, T., Zou, Q.X. \& Zeng, X.H. (2015) Emodin inhibits human sperm functions by reducing sperm $[\mathrm{Ca} 2+]_{\mathrm{i}}$ and tyrosine phosphorylation. Reprod. Toxicol., 51, 14-21.

Makkar, G., Ng, H.Y., Yeung, S.B. \& Ho, P.C. (1999) Comparison of two colloidal silica-based sperm separation media with a non-silica-based medium. Fertil. Steril., 72, 796-802.

Mu, X., Yang, L. \& Chang, C. (2006) Stage dependent and androgen inductive expression of orphan receptor TR4 in rat testis. Biochem. Biophys. Res. Commun., 341, 464-469. 
Olesen, C., Hansen, C., Bendsen, E., Byskov, A.G., Schwinger, E., Lopez-Pajares, I., Jensen, P.K., Kristoffersson, U., Schubert, R., Van Assche, E., Wahlstroem, J., Lespinasse, J. \& Tommerup, N. (2001) Identification of human candidate genes for male infertility by digital differential display. Mol. Hum. Reprod., 7, 11-20.

Parte, P.P., Rao, P., Redij, S., Lobo, V., D’Souza, S.J., Gajbhiye, R. \& Kulkarni, V. (2012) Sperm phosphoproteome profiling by ultra performance liquid chromatography followed by data independent analysis (LC-MS(E)) reveals altered proteomic signatures in asthenozoospermia. J. Proteomics, 75, 58615871.

Peng, S., Yang, J., Chen, H., Guo, X., Li, D., Zhou, H., Gan, Y., Jiang, X. \& Tang, Y. (2012) Construction of TDRG1 shRNA expression vector and interfering effect of TDRG1 shRNA expression vector on NTERA-2 cells. Zhong Nan Da Xue Xue Bao Yi Xue Ban, 37, 979-982.

Perl, A., Qian, Y., Chohan, K.R., Shirley, C.R., Amidon, W., Banerjee, S., Middleton, F.A., Conkrite, K.L., Barcza, M., Gonchoroff, N., Suarez, S.S. \& Banki, K. (2006) Transaldolase is essential for maintenance of the mitochondrial transmembrane potential and fertility of spermatozoa. Proc. Natl. Acad. Sci. USA, 103, 14813-14818.

Poongothai, J., Gopenath, T.S. \& Manonayaki, S. (2009) Genetics of human male infertility. Singapore Med. J., 50, 336-347.

Scheurle, D., DeYoung, M.P., Binninger, D.M., Page, H., Jahanzeb, M. \& Narayanan, R. (2000) Cancer gene discovery using digital differential display. Cancer Res., 60, 4037-4043.

Sharma, R.K., Seifarth, K., Garlak, D. \& Agarwal, A. (1999) Comparison of three sperm preparation media. Int. J. Fertil. Womens Med., 44, 163-167.

Siva, A.B., Kameshwari, D.B., Singh, V., Pavani, K., Sundaram, C.S., Rangaraj, N., Deenadayal, M. \& Shivaji, S. (2010) Proteomics-based study on asthenozoospermia: differential expression of proteasome alpha complex. Mol. Hum. Reprod., 16, 452-462.

Tubbs, C. \& Thomas, P. (2009) Progestin signaling through an olfactory $\mathrm{G}$ protein and membrane progestin receptor-alpha in Atlantic croaker sperm: potential role in induction of sperm hypermotility. Endocrinology, 150, 473-484.

Vadnais, M.L., Lin, A.M. \& Gerton, G.L. (2014) Mitochondrial fusion protein MFN2 interacts with the mitostatin-related protein MNS1 required for mouse sperm flagellar structure and function. Cilia, 3, 5 .

Visconti, P.E., Hao, Z., Purdon, M.A., Stein, P., Balsara, B.R., Testa, J.R., Herr, J.C., Moss, S.B. \& Kopf, G.S. (2001) Cloning and chromosomal localization of a gene encoding a novel serine/threonine kinase belonging to the subfamily of testis-specific kinases. Genomics, 77, 163-170.

Wen, J., Jiang, X., Tang, Y., Yang, J., Chen, H. \& Liu, Z. (2010) Preparation and identification of monoclonal antibody against human testis development related gene 1. Zhong Nan Da Xue Xие Bao Yi Xue Ban, 35, 230-235.

Yang, C., Zeng, X.H., Zhou, Y., Xia, X.M. \& Lingle, C.J. (2011a) LRRC52 (leucine-rich-repeat-containing protein 52), a testisspecific auxiliary subunit of the alkalization-activated Slo3 channel. Proc. Natl. Acad. Sci. USA, 108, 19419-19424.

Yang, S.R., Wang, W.P., Lei, C., Liu, Q.M., Xu, F.Q., Xing, X.W., Chen, H., Liu, J.J., Wu, S.L. \& Wang, M.H. (2011b) Localization and characterization of rat transmembrane protein 225 specifically expressed in testis. DNA Cell Biol., 30, 9-16.

Zeng, X.H., Navarro, B., Xia, X.M., Clapham, D.E. \& Lingle, C.J. (2013) Simultaneous knockout of Slo3 and CatSper1 abolishes all alkalization- and voltage-activated current in mouse spermatozoa. J. Gen. Physiol., 142, 305-313.

Zheng, K., Yang, F. \& Wang, P.J. (2010) Regulation of male fertility by X-linked genes. J. Androl., 31, 79-85. 